\title{
Disseminated Microsporidiosis Due to Encephalitozoon hellem: Pulmonary Colonization, Microhematuria, and Mild Conjunctivitis in a Patient with AIDS
}

\author{
Rainer Weber, Herbert Kuster, Govinda S. Visvesvara, \\ Ralph T. Bryan, David A. Schwartz, and Ruedi Lüthy
}

\author{
From the Division of Infectious Diseases, Department of Medicine, \\ University Hospital, Zurich, Switzerland; and the Division of Parasitic \\ Diseases, Centers for Disease Control and Prevention, and the \\ Department of Pathology, Emory University School of Medicine, \\ Atlanta, Georgia
}

\begin{abstract}
Four genera of microsporidia have been associated with disease in humans, which predominantly affects immunocompromised persons. Systemic infection with a newly characterized microsporidian species, Encephalitozoon hellem, was recently reported in a patient with AIDS. This article describes a second patient with AIDS and disseminated $E$. hellem infection. In this case the parasite was detected in sputum, urine, and conjunctival swab specimens. Apart from recurrent mild conjunctivitis and asymptomatic microhematuria, the patient had no findings or symptoms that could be related to this parasite. Specifically, no microsporidian-associated pulmonary pathology was documented. Detection of $E$. hellem in the patient's sputum may have epidemiological implications in that this finding suggests transmission of microsporidia by the aerosol route. Because the patient died of unrelated complications, it remains unknown whether he was an asymptomatic carrier of microsporidia or whether microhematuria heralded early microsporidian disease, with the onset of cellular damage in the urinary tract.
\end{abstract}

Microsporidia are obligate intracellular protozoal parasites that form spores containing an exceptional extrusion apparatus for injecting the infective sporoplasm into the host cell [1-3]. Their range of hosts includes most invertebrates and all classes of vertebrates [1]. Parasites of the genera Enterocytozoon, Encephalitozoon, Pleistophora, and Nosema as well as still-unclassified microsporidian organisms have been associated with disease in humans, predominantly affecting immunocompromised persons [1-9]. Since 1985, approximately 150 cases of microsporidian infection have been described in patients with AIDS; the majority of these cases have been due to Enterocytozoon bieneusi [3-32].

We recently reported autopsy findings for what we believe to be the first case of disseminated microsporidiosis due to Encephalitozoon hellem in a patient infected with human immunodeficiency virus (HIV). This patient presented with dysuria and gross hematuria. The autopsy showed diffuse interstitial nephritis, tubular necrosis, and diffuse ulcerating cystitis. Microsporidian organisms were found in the urinary tract as well as in bronchial epithelial cells and in corneal epithelial cells, with no inflammatory reaction [27]. This article describes a second case of disseminated infection with $E$. hellem in a patient with AIDS; in this case the parasite was detected in sputum, urine, and conjunctival swab specimens.

\footnotetext{
Received 4 December 1992; revised 25 February 1993.

Reprints or correspondence: Dr. Rainer Weber, Division of Infectious Diseases, Department of Medicine, University Hospital, CH-8091 Zurich, Switzerland.

Clinical Infectious Diseases 1993;17:415-9

(C) 1993 by The University of Chicago. All rights reserved.

1058-4838/93/1702-0014\$02.00
}

Apart from recurrent mild conjunctivitis and asymptomatic microhematuria, the patient had no findings or symptoms that could be associated with microsporidiosis. He died of unrelated complications; thus it is unclear whether he was an asymptomatic carrier of $E$. hellem or whether he had earlystage microsporidian disease.

\section{Case Report}

\section{Description of the Case}

A 49-year-old Swiss man with a history of iv drug use between 1970 and 1984 consulted his primary-care physician in December 1990 with small, nonspecific penile ulcers and a cough productive of scant, nonpurulent sputum. The patient had had acute hepatitis associated with the injection of illicit iv drugs and had developed self-limited fever after a trip to South America in 1989. He had no pets.

In January 1991, he was referred to our HIV outpatient clinic for counseling because antibodies to HIV had been detected in blood collected the previous month. Findings on physical examination were normal apart from oral candidiasis. No penile lesions were evident. The patient's weight was $93 \mathrm{~kg}$; he was $180 \mathrm{~cm}$ tall. The hematocrit was $42.3 \%$. The leukocyte count was $3.4 \times 10^{9} / \mathrm{L}$, with $0.12 \times 10^{9} \mathrm{CD} 4$ cells/ L. Levels of serum creatinine, liver enzymes, and lactate dehydrogenase were normal, as were the results of urinalysis. VDRL and FTA-ABS (fluorescent treponemal antibodyabsorbed) tests gave negative results. Assays for IgG antibodies to hepatitis A virus, hepatitis B core antigen, hepatitis C virus, Toxoplasma, and cytomegalovirus were positive. Chest roentgenography was normal. Antiretroviral therapy (zido- 


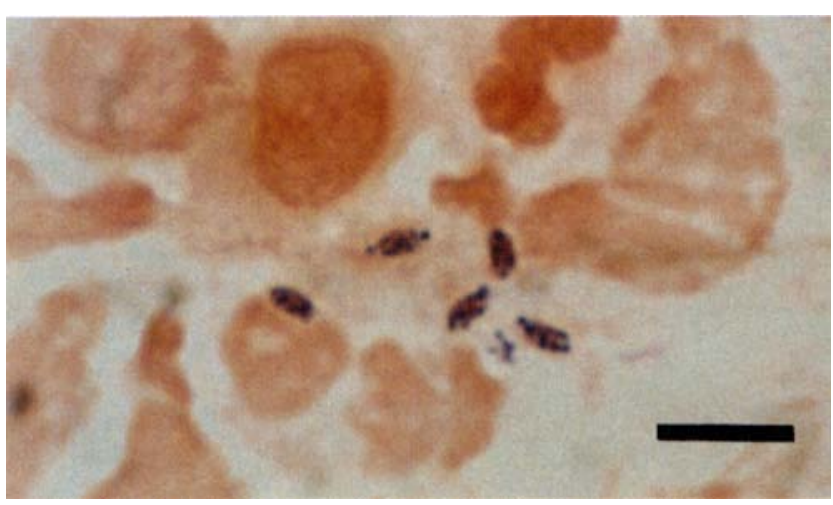

Figure 1. Gram-stained smear of sputum showing microsporidian spores. Bar $=5 \mu \mathrm{m}$.

vudine, $250 \mathrm{mg}$ twice daily), primary prophylaxis for Pneumocystis carinii pneumonia (aerosolized pentamidine, 300 $\mathrm{mg}$ monthly), and treatment of oral candidiasis (fluconazole) were started.

Beginning in January 1991, the patient had recurrent episodes of mild bilateral conjunctivitis ("red eyes") that resolved without treatment. In July, ophthalmic herpes zoster of the left eye was treated with acyclovir. In August, cytomegalovirus chorioretinitis of the right eye was treated with ganciclovir, after which maintenance therapy was begun. At that point the antiretroviral regimen was changed from zidovudine to dideoxyinosine. In February 1992, catheter-related Staphylococcus aureus septicemia and consecutive septic lung abscesses were treated with iv flucloxacillin.

In May 1992, the patient presented with dyspnea, cough, and fever. He had a temperature of $38.0^{\circ} \mathrm{C}$. Physical examination was unrevealing. The patient weighed $84 \mathrm{~kg}$. The hematocrit was $31.6 \%$; the leukocyte count was $2.58 \times 10^{9} / \mathrm{L}$, with $0.05 \times 10^{9} \mathrm{CD} 4$ cells/L. Concentrations of serum creatinine, liver enzymes, alkaline phosphatase, and lactate dehydrogenase were normal. Chest roentgenography showed multiple small pulmonary abscesses. Cultures of both blood and sputum were positive for $S$. aureus. Echocardiography was normal. The patient was treated with iv amoxicillin/ clavulanate, and his condition improved.

Pulmonary symptoms were evaluated at this time. Examination of sputum by light microscopy revealed no P. carinii but did detect gram-positive cocci as well as intracellular and extracellular microsporidian organisms measuring 1.0-1.3 $\mu \mathrm{m}$ by $2.0-3.2 \mu \mathrm{m}$ (figure 1). The results of urinalysis were normal, but microsporidian spores were seen in smears of urine sediment obtained by centrifugation at $1,500 \mathrm{~g}$ (figure 2). Although the patient had no conjunctivitis at this time, microscopic examination of conjunctival swabs showed microsporidian spores intracellularly and extracellularly (figures 3 and 4).

In June 1992, the patient was again hospitalized because

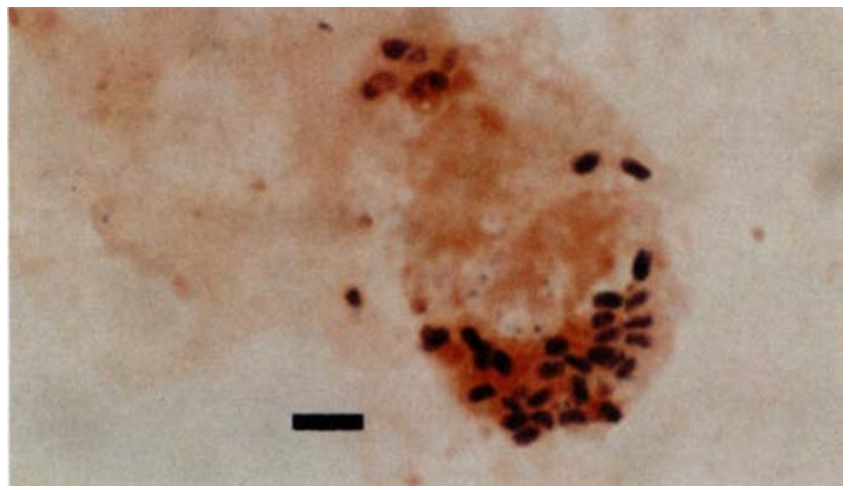

Figure 2. Gram-stained smear of urine sediment showing intracellular microsporidian spores. Bar $=5 \mu \mathrm{m}$.

of staphylococcal septicemia and pulmonary abscesses. He again responded to treatment with appropriate iv antibiotics. Repeated examinations of sputum, urine, and conjunctival swab samples showed microsporidian spores. No microsporidia were found in stool specimens examined by previously described techniques [25]. The patient never had diarrhea. The CD4 cell count dropped to $0.01 \times 10^{9} / \mathrm{L}$. The serum creatinine level was normal, but urinalysis now revealed mild proteinuria, leukocytes, erythrocytes, and hyaline casts.

The patient died of Escherichia coli septicemia in July 1992. Permission for an autopsy was denied.

\section{Identification of Microsporidian Organisms}

Light microscopy. Smears of washed sputum, conjunctival swab samples, and urine sediment obtained by centrifugation at $1,500 \mathrm{~g}$ were subjected to Giemsa, chromotropebased Weber, and gram staining (as previously described [25]) and to an indirect fluorescein-tagged antibody immunofluorescence (IIF) assay for $P$. carinii. The smears were then

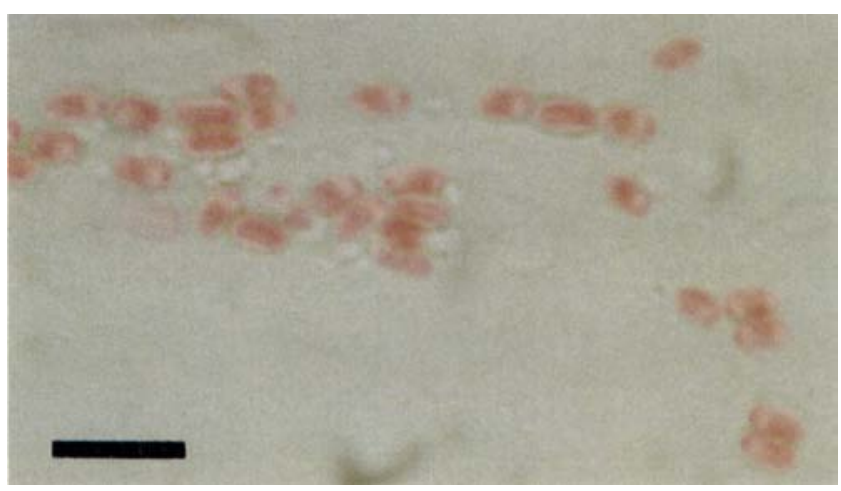

Figure 3. Conjunctival swab sample showing pinkish-red-staining microsporidian spores (chromotrope-based Weber stain). Bar $=$ $5 \mu \mathrm{m}$. 


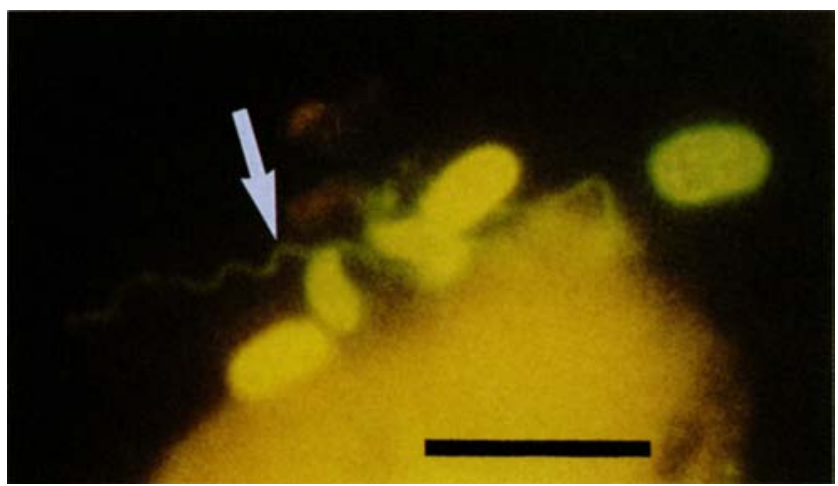

Figure 4. Immunofluorescence staining of a conjunctival swab sample showing $E$. hellem spores. A slender polar tubule extrudes from one spore (arrow). Bar $=5 \mu \mathrm{m}$.

examined at magnifications of $\times 630$ and $\times 1,000$. The organisms detected were suspected to be microsporidian spores because of their oval shape, intracellular location, size, and staining pattern [25]. Smears stained according to the chromotrope-based Weber technique were found to include ovoid and refractile microsporidian spores whose walls stained bright pinkish-red (figure 3 ). Most spores had a distinct, pinkish-red-staining, belt-like stripe girding them diagonally or equatorially. Gram staining revealed either partially gram-positive microsporidian spores (figure 2) or gram-negative spores with gram-positive dots (figure 1).

Electron microscopy. The presence of spores with characteristics typical of microsporidia was confirmed by examination of sputum and urine sediment (figures 5-7). The microsporidian genus Encephalitozoon was identified mor-

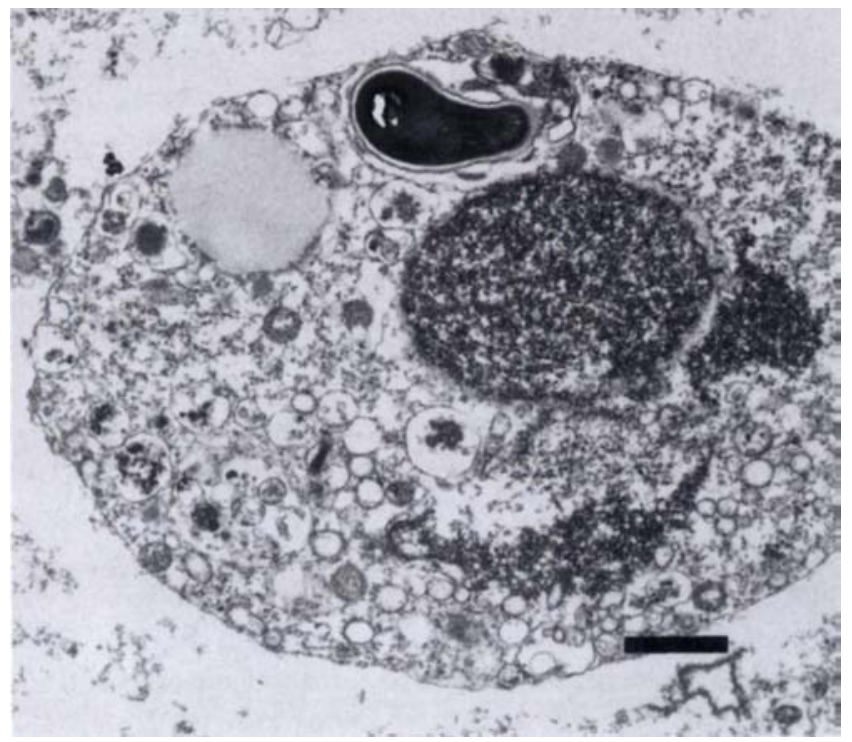

Figure 5. Transmission electron micrograph of sputum showing an intracellular microsporidian spore. $\mathrm{Bar}=1 \mu \mathrm{m}$.

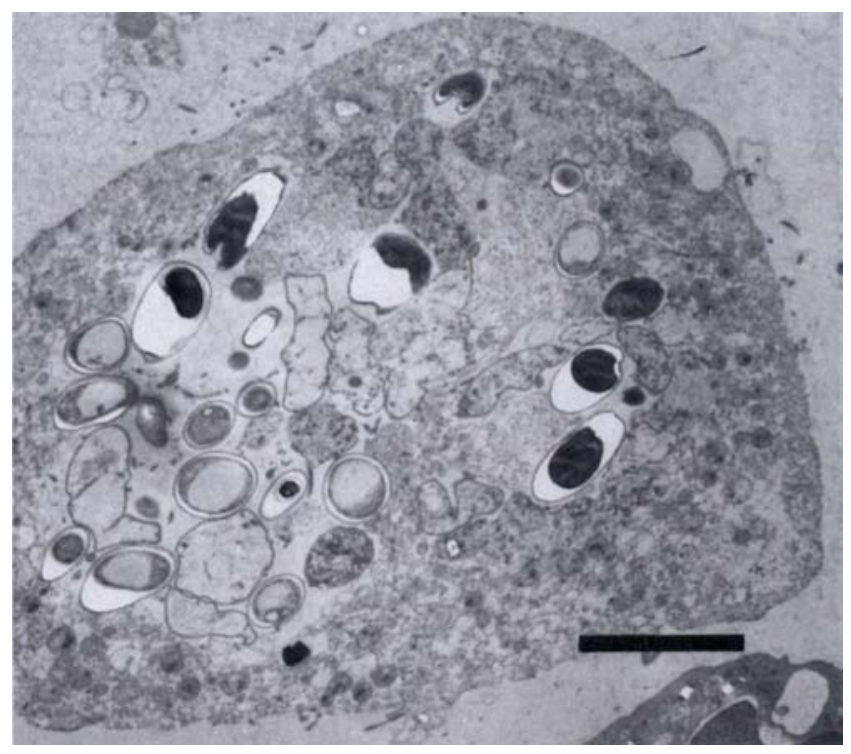

Figure 6. Transmission electron micrograph of urine sediment showing intracellular microsporidian spores. Bar $=5 \mu \mathrm{m}$.

phologically [1]. For electron microscopy, both urine and washed sputum were centrifuged at $500 \mathrm{~g}$ for 10 minutes. The pellet was fixed for 30 minutes at $4{ }^{\circ} \mathrm{C}$ with $3 \%$ glutaraldehyde in PBS and then postfixed for 2 hours at $4^{\circ} \mathrm{C}$ with $2 \%$ osmium tetroxide in $0.1 M$ cacodylate buffer ( $\mathrm{pH} 7.2$ ). After incubation for 12 hours in a $2 \%$ aqueous solution of uranyl acetate, the material was dehydrated with dimethoxypro-

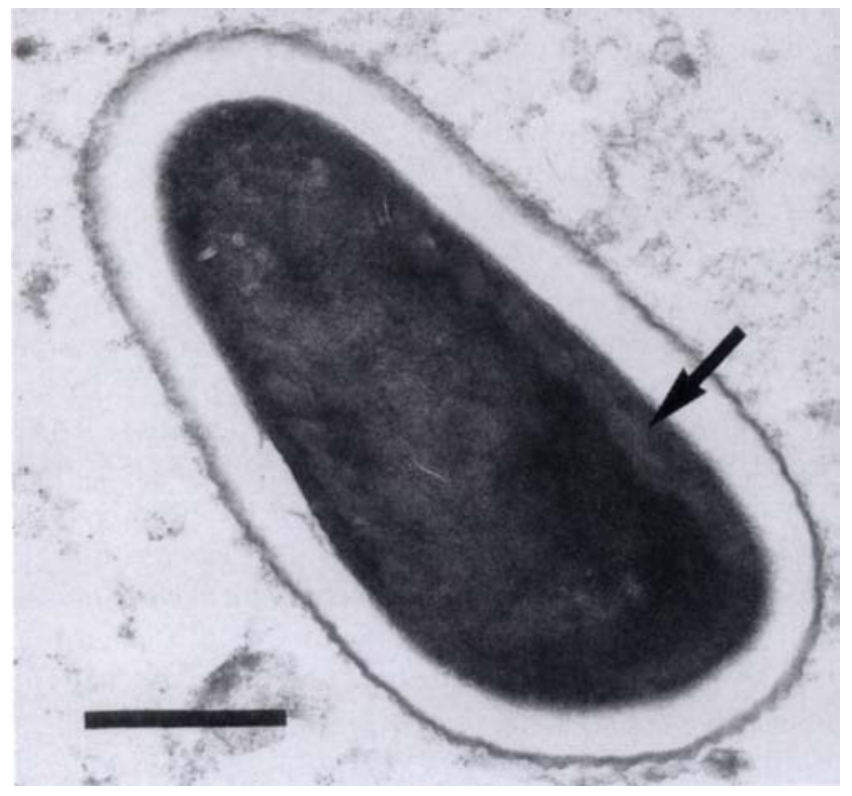

Figure 7. Transmission electron micrograph of an individual microsporidian spore. The spore is characterized by polar tubes (arrow), an electron-lucent endospore layer, and a dense outer coat. Bar $=0.5 \mu \mathrm{m}$. 
pane and embedded in Epon 812 epoxy resin mixture (Fluka Chemie, Buchs, Switzerland). The sections were stained with lead citrate and examined with a transmission electron microscope (model EM 400 HM; Philips Electronics, Eindhoven, the Netherlands).

Antigenic analysis. The parasites in smears of our patient's urine and conjunctival swab specimens were identified to the species level by an IIF technique, as detailed elsewhere [24, 27] (figure 4). In brief, antibody to microsporidian spores cultured from the urine of a previously described patient was produced in rabbits. These cultured parasites had been designated as CDC:0291:V213. Comparison of the reactivity patterns for CDC:0291:V213, E. hellem (obtained from Dr. E. Didier, Covington, LA), and Encephalitozoon cuniculi (obtained from Dr. J. Shadduck, College Station, TX) in the IIF test showed that CDC:O291:V213 was cospecific with $E$. hellem [27]. The antibody produced in rabbits was rendered specific for $E$. hellem by cross-absorption experiments described earlier [27]. Spores from our patient reacted with the $E$. hellem-specific antibody; this result indicated that our patient was infected with $E$. hellem.

In vitro cultivation. Microsporidian spores concentrated from our patient's urine were inoculated into monolayers of monkey kidney (E6) cells, as previously described [24, 27]. Foci of microsporidian infection were identified.

\section{Discussion}

Microsporidia have received increasing attention as opportunistic pathogens in the evolving AIDS pandemic [2, 12 , 13]. One microsporidian, E. bieneusi, has been recognized as a frequent cause of chronic diarrhea in severely immunosuppressed patients with AIDS [7, 8, 20, 22, 25]. Another microsporidian, E. cuniculi, was found in liver biopsy specimens from a patient with AIDS and fulminant hepatitis [10] and in the omentum of an HIV-infected patient with peritonitis [11]. Several cases of HIV-associated microsporidian keratoconjunctivitis have also been reported $[15-18,21,23,27$, $28,30,31]$. Whereas the responsible ocular microsporidia were often identified as $E$. cuniculi, Didier et al. recently described a new species, $E$. hellem, infecting three patients with AIDS who presented with keratoconjunctivitis [17]. Dissemination of $E$. hellem into the urinary, respiratory, and ocular tissues of one patient with AIDS has been reported [27]. An Encephalitozoon species has also been identified in the nasal epithelium of an HIV-infected patient with sinusitis [30]. Most recently, still-unclassified microsporidian organisms have been detected in the small intestine, bile duct, and urinary tract of patients with AIDS [5, 6].

The two known species of Encephalitozoon-E. cuniculi and $E$. hellem-have only recently been distinguished; because they are morphologically identical, antigenic and biochemical analyses are required for their differentiation [17, $24,27]$. In the patient described herein, microsporidia were first detected in a gram-stained smear of sputum. This examination could not readily distinguish microsporidian spores from bacteria, but the organisms were thought to be microsporidia because of their ovoid shape, intracellular location, and size. Smears stained by the chromotrope-based Weber technique included microorganisms that had the same staining pattern as $E$. bieneusi [25] but that were larger. Since we were aware of the dissemination of microsporidian parasites in another patient [27], we examined urine specimens and conjunctival swab samples, which were positive for microsporidia, and stool specimens, which were negative. Electron microscopy and antigenic analysis of the parasite led to diagnostic confirmation of infection with $E$. hellem.

Neither the source of microsporidia infecting humans nor their mode of transmission is known. In microsporidiosis of mammals (e.g., infection with E. cuniculi), the primary site of infection is usually the small-intestinal enterocyte. Thereafter, the parasites infect endothelial cells and macrophages and thus are dispersed to various other organs (including the lungs), showing a predilection for the brain and the kidneys [1]. Infection by the oral route has been documented experimentally [33].

The possible transmission of microsporidia by the aerosol route is indicated by the case of our patient, who repeatedly expectorated microsporidian spores in his sputum; by autopsy findings in another case, in which numerous $E$. hellem parasites were found in the lining epithelium of the tracheobronchial tree [27]; and by a case of pulmonary infection due to E. bieneusi [32]. Pulmonary infections have been induced experimentally by instillation of E. cuniculi spores into the nasal cavities as well as by intratracheal injection of $E$. cuniculi spores into rabbits $[1,33]$.

The clinical spectrum of human microsporidiosis has yet to be fully evaluated. The case reported here and another described previously [27] suggest that infection with $E$. hellem tends to disseminate and has a predilection for the kidneys, the tracheobronchial tree, and the conjunctiva. In contrast, infection with $E$. bieneusi appears to disseminate rarely, if ever $[20,32]$.

Pulmonary pathology probably was not associated with the $E$. hellem organisms detected in sputum specimens from our patient. Whether microhematuria heralded early microsporidian disease, with the onset of cellular damage in the urinary tract, remains unknown. However, gross hematuria and renal failure were early signs of disseminated $E$. hellem infection in the previously described patient; in that instance, autopsy showed diffuse interstitial nephritis, tubular necrosis, ureteritis, and diffuse ulcerating cystitis [27]. The first microsporidium-associated symptoms in the patient described herein were ocular. Unlike previously reported cases of HIV-associated ocular encephalitozoon microsporidiosis manifesting with epithelial keratopathy [15-18, 21, 23, 27, $28,30,31]$, our patient's recurrent episodes of "red eyes" resolved without treatment. 
Our patient may reflect a case of virtually asymptomatic microsporidian carriage. It is not known whether he had an early stage of microsporidian disease or whether encephalitozoon infection per se is not very pathogenic. Like other opportunistic infections, such as those due to Mycobacterium avium-Mycobacterium intracellulare or cytomegalovirus, disseminated microsporidiosis due to $E$. hellem may occur primarily in patients with very low CD4 cell counts.

\section{Acknowledgments}

The authors thank Professor Thomas Bächi, Ph.D., and Ruth Keller (Laboratory of Electron Microscopy, University of $\mathrm{Zu}$ rich); and Bärbel Sauer (Laboratory of Infectious Diseases, University Hospital, Zurich) for technical support. They also thank Sandra Opravil for editorial assistance.

\section{References}

1. Canning EU, Lom J, Dykova I. The microsporidia of vertebrates. New York: Academic Press, 1986.

2. Bryan RT. Microsporidia. In: Mandell GL, Douglas RG. Bennett JE, eds. Principles and practice of infectious diseases. 3rd ed. New York: Churchill Livingstone, 1990:2130-4.

3. Bryan RT, Cali A, Owen RL. Spencer HC. Microsporidia: opportunistic pathogens in patients with AIDS. In: Sun T, ed. Progress in clinical parasitology. Vol 2. Philadelphia: Field and Wood, 1991:1-26.

4. Cali A, Orenstein JM, Kotler DP, Owen R. A comparison of two microsporidian parasites in enterocytes of AIDS patients with chronic diarrhea. J Protozool 1991;38(suppl):96S-8S.

5. Orenstein JM, Tenner M. Cali A, Kotler DP. A microsporidian previously undescribed in humans, infecting enterocytes and macrophages. and associated with diarrhea in an acquired immunodeficiency syndrome patient. Hum Pathol 1992;23:722-8.

6. Orenstein JM. Dieterich DT, Kotler DP. Systemic dissemination by a newly recognized intestinal microsporidia species in AIDS. AIDS 1992;6:1143-50.

7. Dobbins WO III, Weinstein WM. Electron microscopy of the intestine and rectum in acquired immunodeficiency syndrome. Gastroenterology 1985;88:738-49.

8. Modigliani R, Bories C, Le Charpentier Y, et al. Diarrhoea and malabsorption in acquired immune deficiency syndrome: a study of four cases with special emphasis on opportunistic protozoan infections. Gut 1985;26:179-87.

9. Ledford DK, Overman MD, Gonzalvo A, Cali A, Mester SW, Lockey RF. Microsporidiosis myositis in a patient with the acquired immunodeficiency syndrome. Ann Intern Med 1985;102:628-30.

10. Terada S, Reddy KR, Jeffers LJ, Cali A, Schiff ER. Microsporidian hepatitis in the acquired immunodeficiency syndrome. Ann Intern Med 1987;107:61-2.

11. Zender HO, Arrigoni E, Eckert J, Kapanci Y. A case of Encephalitozoon cuniculi peritonitis in a patient with AIDS. Am J Clin Pathol 1989;92:352-6.

12. Shadduck JA. Human microsporidiosis and AIDS. Rev Infect Dis 1989;11:203-7.

13. Canning EU, Hollister WS. Enterocytozoon bieneusi (Microspora): prevalence and pathogenicity in AIDS patients. Trans $R$ Soc Trop Med Hyg 1990;84:181-6.
14. Cali A, Owen RL. Intracellular development of Enterocytozoon, unique microsporidian found in the intestine of AIDS patients. J Protozool 1990;37:145-55.

15. Centers for Disease Control. Microsporidian keratoconjunctivitis in patients with AIDS. Morb Mortal Wkly Rep 1990;39:188-9.

16. Davis RM, Font RL, Keisler MS, Shadduck JA. Corneal microsporidiosis. A case report including ultrastructural observations. Ophthalmology 1990;97:953-7.

17. Didier ES, Didier PJ, Friedberg DN, et al. Isolation and characterization of a new human microsporidian, Encephalitozoon hellem (n.sp.), from three AIDS patients with keratoconjunctivitis. J Infect Dis 1990;163:617-21.

18. Friedberg DN, Stenson SM, Orenstein JM, Tierno PM, Charles NC. Microsporidial keratoconjunctivitis in acquired immunodeficiency syndrome. Arch Ophthalmol 1990;108:504-8.

19. Kotler DP, Francisco A, Clayton F, Scholes JV, Orenstein JM. Small intestinal injury and parasitic diseases in AIDS. Ann Intern Med 1990;113:444-9.

20. Orenstein JM, Chiang J, Steinberg W, Smith PD, Rotterdam H, Kotler DP. Intestinal microsporidiosis as a cause of diarrhea in human immunodeficiency virus-infected patients: a report of 20 cases. Hum Pathol 1990;21:475-81.

21. Cali A, Meisler DM, Lowder CY, et al. Corneal microsporidioses: characterization and identification. J Protozool 1991;38(suppl):215S$7 \mathrm{~S}$.

22. Eeftinck Schattenkerk JKM, van Gool T, van Ketel RJ, et al. Clinical significance of small-intestinal microsporidiosis in HIV-1-infected individuals. Lancet 1991;337:895-8.

23. Cali A, Meisler DM, Rutherford I, et al. Corneal microsporidiosis in a patient with AIDS. Am J Trop Med Hyg 1991;44:463-8.

24. Visvesvara GS, Leitch GJ, Moura H, Wallace S, Weber R, Bryan RT. Culture, electron microscopy, and immunoblot studies on a microsporidian parasite isolated from the urine of a patient with AIDS. $\mathrm{J}$ Protozool 1991;38(suppl): 105S-1 IS.

25. Weber R, Bryan RT, Owen RL, et al. Improved light-microscopical detection of microsporidia spores in stool and duodenal aspirates. $\mathrm{N}$ Engl J Med 1992;326:161-6.

26. Orenstein JM, Tenner M, Kotler DP. Localization of infection by the microsporidian Enterocytozoon bieneusi in the gastrointestinal tract of AIDS patients with diarrhea. AIDS 1992;6:195-7.

27. Schwartz DA, Bryan RT. Hewan-Lowe KO, et al. Disseminated microsporidiosis (Encephalitozoon hellem) and acquired immunodeficiency syndrome. Arch Pathol Lab Med 1992;1 16:660-8.

28. Canning EU, Curry A, Lacey CJ, Fenwick D. Ultrastructure of Encephalitozoon sp. infecting the conjunctival, corneal and nasal epithelia of a patient with AIDS. European Journal of Protistology 1992; 28:226-37.

29. Blanshard C, Hollister WS, Peacock CS, et al. Simultaneous infection with two types of intestinal microsporidia in a patient with AIDS. Gut 1992;33:418-20.

30. Lacey CJ. Clark AM, Fraser P, Metcalfe T, Bonsor G, Curry A. Chronic microsporidian infection of the nasal mucosae, sinuses and conjunctivae in HIV disease. Genitourin Med 1992;68:179-81.

31. Metcalfe TW, Doran RML, Rowland PL, Curry A, Lacey CJN. Microsporidial keratoconjunctivitis in a patient with AIDS. $\mathrm{Br} \mathrm{J}$ Ophthalmol 1992;76:177-8.

32. Weber R, Kuster H, Keller R, et al. Pulmonary and intestinal microsporidiosis in a patient with the acquired immunodeficiency syndrome. Am Rev Respir Dis 1992;146:1603-5.

33. Cox JC, Hamilton RC, Attwood HD. An investigation of the route and progression of Encephalitozoon cuniculi infection in adult rabbits. J Protozool 1979;26:260-5. 